\title{
APPLICATION OF GRAY TARGET MODELS IN THE PREDICTION OF COAL AND GAS OUTBURST: THE CASE OF JINZHUSHAN COAL MINE IN CHINA
}

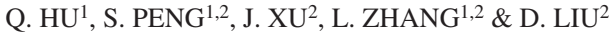 \\ ${ }^{1}$ Chongqing Research Institute Co. Ltd. of China Coal Technology \& Engineering Group Corporation, China. \\ ${ }^{2}$ State Key Laboratory of Coal Mine Disaster Dynamics and Control, Chongqing University, China.
}

\begin{abstract}
Coal and gas outbursts in coal mines are a complex dynamic geological phenomenon. A gray target model has been established based on the gray system theory to predict coal and gas outbursts. The model considers four influencing factors for coal and gas outbursts: gas pressure, destructive type of coal, coal rigidity, and initial speed of methane diffusion. Each weight of the factors is given through an improved analytic hierarchy process without consistency checks, and the accuracy of the assessment is high. By using the model, coal and gas outbursts in the Jinzhushan mine were predicted. Results demonstrate the viability of the gray target model in the prediction of coal and gas outbursts.

Keywords: Coal and gas outburst, gray target model, prediction, relational degree.
\end{abstract}

\section{INTRODUCTION}

Coal and gas outbursts in coal mines represent a complex dynamic geological phenomenon. Many coal and gas emissions have occurred, from coal mines to highways, in a short period of time. These outbursts have great effects, and they are a particularly serious threat to the safety of coal mine production. Gas outbursts in China as a whole are a serious threat. With the development of the coal industry, new mining areas are being developed and old mines are being extended. Consequently, gas outbursts have been occurring more frequently in coal mines. As the mining scale expands, the number of gas outbursts consequently increases. The purpose of having coal and gas outburst predictions or forecasts is to determine whether reasonable and effective measures could be taken to prevent sudden occurrences, to provide a scientific basis, to reduce anti-outburst engineering works and the time needed to ensure that the normal conduct of mining production is maintained, and finally to protect lives and property safety underground. Forecast and control of coal and gas outbursts are important because they increase the social and economic benefits of mines.

The impact of coal and gas outburst is complex and dependent on a number of factors, such as geological, artificial, regional, partial, definite, and uncertainty. Based on existing research results, certain factors or indicators can be difficult to identify. Coal and gas outbursts also do not always show direct quantitative relationships with these factors, so that forecasting them can be difficult. In existing studies, a variety of methods have been used to evaluate coal and gas outbursts, such as fuzzy synthetic evaluation, neural network evaluation, and extension clustering [1-3], to determine measures to reduce the loss induced by sudden occurrences [4].

However, conventional deterministic methods of geotechnical analysis need to be supplemented by studies that use a probabilistic framework that considers parameter variability and other uncertainties [5-10]. The gray target theory is an important part of risk assessment methodology to analyze the multi-index control factors of coal and gas outbursts [11-13]. In this study, a gray target model for the prediction of coal and gas outburst is established, and the case study of the Jinzhushan coal mine is considered to validate its application results. 


\section{METHODS}

\subsection{Gray target theory}

Gray target is a newly developed theory in gray systems. Gray patterns are a part of gray evaluation and gray decision-making process recently proposed by Julong [14]. Grades determined by gray evaluation reflect objectively the integrated consequence of interaction and impact of all factors in a system. In gray target analysis, the reference series, termed the standard mode is not known but is constructed from the series to be analyzed.

The basic procedure of gray target modeling includes four stages:

1. Construction of the standard pattern by using the data approaching the target value of the sub-proposition. The sub-proposition here consists of the evaluation factors or properties of a decision making.

2. Construction of a gray target with all patterns being analyzed and the standard pattern; thus, the standard pattern becomes the 'bull's-eye' of the gray target.

3. Comparison of the patterns being analyzed with the standard pattern, and calculation of the gray target coefficients and the approaching degree.

4. Determination of the evaluation grade of every pattern according to the results and reference grades.

The patterns, apart from the 'bull's-eye', are named gray target edges. The top mode relative to the 'bull's-eye' is labeled as the upper edge of the gray target, whereas the bottom mode is called the lower edge of the gray target. The gray relational degree of the bull's-eye and of every pattern in gray relational information difference space is termed the approaching degree $[15,16]$.

\subsection{Establishment of a sample matrix}

Suppose $n$ objects or $n$ decision makings of schemes $E_{i}$ are assessed. Schemes here are the multiple attribute decision problems such as assessment of the possibility of coal and gas outbursts. These objects or decision makings of the schemes constitute a decision scheme set $E, E=\left\{E_{1}, E_{2}, \ldots, E_{n}\right\}$ with $m$ associated evaluation factors or properties, which constitute the factor set $A, A=\left\{A_{1}, A_{2}, \ldots, A_{m}\right\}$. The effect sample value of scheme $E_{i}$ for factor $A_{j}$ is $x_{i j}$ $(i=1,2, \ldots, n ; j=1,2, \ldots, m)$. The effect sample matrix of the decision scheme set $E$ is as follows:

$$
X=\left[\begin{array}{cccc}
x_{11} & x_{12} & \cdots & x_{1 m} \\
x_{21} & x_{22} & \cdots & x_{2 m} \\
\vdots & \vdots & \vdots & \vdots \\
x_{n 1} & x_{n 2} & \cdots & x_{n m}
\end{array}\right] .
$$

\subsection{Establishment of the decision matrix}

Generally, the factor attribute of set A can be divided into three types: benefit, cost, and interval. As the indicators of centralized factors have different dimensions, the original effect of the sample matrix needs initialization. Considering the shortcomings of the $[0,1]$ interval generation method for data sequence, a linear transformation operator $[-1,1]$ is used to trans- 
form the effect sample matrix $X$ based on fuzzy set pair analysis [17]. This is a modified uncertainty theory transformation regarding both the certain side and the uncertain side. The core of this uncertainty theory is to consider certainties and uncertainties as a certain-uncertain system by studying the relationship between the certainty and the uncertainty sides of the problems from three aspects of identity, discrepancy, and contrariness.

Assume that $Z_{(k)}$ is the average value of the effect sample value $x_{i j}$. It is defined as follows:

$$
Z_{(k)}=\frac{1}{m} \sum_{i=1}^{m} x_{i(k)}(k=1,2, \ldots, n)
$$

Then, the benefit index can be calculated as follows:

$$
\mathrm{r}_{i(k)}=\frac{x_{i(k)}-Z_{(k)}}{\max \left(\max _{i}\left\{x_{i(k)}\right\}-Z_{(k)}, Z_{(k)}-\min _{i}\left\{x_{i(k)}\right\}\right)} \quad(i=1,2, \ldots, m) .
$$

The cost index is calculated as follows:

$$
\mathrm{r}_{i(k)}=\frac{Z_{(k)}-x_{i(k)}}{\max \left(\max _{i}\left\{x_{i(k)}\right\}-Z_{(k)}, Z_{(k)}-\min _{i}\left\{x_{i(k)}\right\}\right)} \quad(i=1,2, \ldots, m) .
$$

The decision matrix $R$ is calculated as follows:

$$
R=\left[\begin{array}{cccc}
r_{11} & r_{12} & \cdots & r_{1 m} \\
r_{21} & r_{22} & \cdots & r_{2 m} \\
\vdots & \vdots & \vdots & \vdots \\
r_{n 1} & r_{n 2} & \cdots & r_{n m}
\end{array}\right]=\left(r_{1}, r_{2}, \cdots, r_{n}\right)
$$

The optimum effect vector of the multiple indicator gray targets is defined as the bull'seye, which is generally expressed as follows:

$$
r_{i}^{0}=\max \left\{x_{\mathrm{ij}}\right\} \quad(1 \leq i \leq n, j=1,2, \ldots, m) .
$$

To calculate the distance between the factor data and the critical factor value of a coal and gas outburst, the critical factor value is denoted as the optimal effect vector $r$.

\subsection{Determining the factor weight based on an improved analytic hierarchy process}

In the analysis, the impact of factors has a direct relationship with the outcome. However, testing the consistency of the decision matrix $R$ is usually difficult. The improved analytic hierarchy process (AHP) [18] not only tests the consistency of the judgment matrix but also assists in determining convergence speed. In addition, the weights of the influencing factors obtained by using this method are more consistent with reality. In this study, the weight $\omega$ of the coal and gas outburst factors is obtained directly through the improved AHP:

$$
\omega=\left(\omega_{1}, \omega_{2}, \cdots, \omega_{m}\right) \quad\left(\omega_{i}>0, i=1,2, \ldots, m\right) .
$$


where $\omega_{i}$ is the distribution ratio of the $r_{i}$ factor for the division, and it should meet the following equation:

$$
\sum_{i=1}^{m} \omega_{i}=1
$$

2.5 Calculation of the off-target distance

The off-target distance of the effect vector is calculated as follows:

$$
\mathrm{e}_{i}=\left|r_{i}-r\right|=\sqrt{\omega_{1}\left(r_{i 1}-r_{1}^{0}\right)^{2}+\omega_{2}\left(r_{i 2}-r_{2}^{0}\right)^{2}+\cdots+\omega_{m}\left(r_{i m}-r_{m}^{0}\right)^{2}} .
$$

The smaller off-target distance $e_{i}$ of the effect vector $r_{i}$ leads to a better decision scheme $E_{i}$. By contrast, the worse decision scheme $E_{i}$ is usually induced by a larger off-target distance $e_{i}$ of the effect vector $r_{i}$.

\section{RESULTS AND DISCUSSION}

Coal and gas outbursts represent a complex and dynamic process, and their causal factors vary from region to region. In the current studies, the widely accepted hypothesis on the coal and gas outbursts is termed the comprehensive hypothesis. This assumes that coal and gas outbursts are regarded as the result of earth stress, gas pressure, and the physical and mechanical properties of coal $[19,20]$. Accordingly, the four factors selected in this study are gas pressure $P$ (represented by $X_{1}$ ), type of coal damage $D$ (represented by $X_{2}$ ), coal rigidity $f$ (represented by $X_{3}$ ), and initial speed of methane diffusion $\Delta P$ (represented by $X_{4}$ ).

The data measured for the four parameters from the Jinzhushan coal mine [22] are used to establish a gray target model. The data are shown in Table 1. According to eqns (3) and (4), the matrix $R$ is shown in Table 2 .

To calculate the distance between the data of each index and the critical value, the critical value is selected as the optimal effect vector $r_{\mathrm{i}}^{0}=(0.074233,0.020833,0.081560$, and 0.022634). Based on the improved AHP, the weights $\omega$ of the coal and gas outburst factor $\omega=(0.45,0.10,0.28$, and 0.17$)$. The off-target distance of the effect vector, according to eqn (9), is shown in Table 3.

From the 10 measure stations data available of coal and gas outburst area and off-target distance, a prediction of coal seam hazard single index critical value was obtained. The outburst hazard degree was divided into four degrees as done in Liang et al. [13] and is shown in Table 4, as small, general, large, and larger depending on the value of the off-target distance. The results of the off-target distance show that all of the predicted points in the Jinzhushan coal mine have a high risk of coal and gas outburst. Coal and gas outburst occurrence is the synthesized result of ground stress, gas pressure, and physical and mechanical characteristics due to the coupling interaction between coal and gas. As shown in Table 1, the gas pressure is almost above the critical value. The early speed of gas emission is high, the coal structure is strongly undermined, the strength is poor, and the concentration of stress is extreme. In theory, non-uniform distribution of in situ stress, high gas pressure and low permeability, and deformation and damage in the soft coal are the favorable conditions for coal and gas outbursts. Therefore, from the view of theoretical analysis, the Jinzhushan coal mine has a high risk of coal and gas outbursts. In the coal mining process, the following must be observed: gas emission at the coal face, changes in the coal destruction type, geological 
Table 1: Measured values of the four factors.

\begin{tabular}{lcccc}
\hline Number & $\begin{array}{c}\text { Gas pressure } P \\
(\mathrm{MPa})\end{array}$ & $\begin{array}{c}\text { Type of coal } \\
\text { damage } D\end{array}$ & $\begin{array}{c}\text { Coal } \\
\text { rigidity } f\end{array}$ & $\begin{array}{c}\text { Initial speed } \\
\text { of methane } \\
\text { diffusion } \Delta P\end{array}$ \\
\hline 1 & 1.20 & 3 & 0.16 & 18 \\
2 & 0.97 & 5 & 0.24 & 6 \\
3 & 2.98 & 3 & 0.24 & 14 \\
4 & 1.80 & 3 & 0.59 & 8 \\
5 & 0.49 & 3 & 0.28 & 11 \\
6 & 1.16 & 4 & 0.34 & 14 \\
Critical value & 0.74 & 3 & 0.50 & 10 \\
\hline
\end{tabular}

Table 2: Matrix R.

\begin{tabular}{lcrrr}
\hline Number & $X_{1}$ & \multicolumn{1}{c}{$X_{2}$} & \multicolumn{1}{c}{$X_{3}$} & \multicolumn{1}{c}{$X_{4}$} \\
\hline 1 & 0.016774 & 0.020833 & -0.087230 & -0.092590 \\
2 & 0.045503 & -0.076390 & -0.047520 & 0.080247 \\
3 & -0.205570 & 0.020833 & -0.047520 & -0.034980 \\
4 & -0.058170 & 0.020833 & 0.126241 & 0.051440 \\
5 & 0.105460 & 0.020833 & -0.027660 & 0.008230 \\
6 & 0.021770 & -0.027780 & 0.002128 & -0.034980 \\
Critical value & 0.074233 & 0.020833 & 0.081560 & 0.022634 \\
\hline
\end{tabular}

Table 3: Result of the off-target distances.

\begin{tabular}{lcccccc}
\hline Number & 1 & 2 & 3 & 4 & 5 & 6 \\
\hline$E$ & 0.11 & 0.08 & 0.20 & 0.09 & 0.06 & 0.06 \\
\hline
\end{tabular}

Table 4: Division of outburst hazard degree types [13].

\begin{tabular}{lcccc}
\hline The degree of risk & Larger & Large & General & Small \\
\hline$E$ & $(0,0.3]$ & $(0.3,0.5]$ & $(0.5,0.7]$ & $>0.7$ \\
\hline
\end{tabular}

structure, and signs of coal and gas outburst. Protective measures to prevent coal and gas outburst must be taken at the same time [21].

The prediction results are consistent with those from the neural network method as well as the actual situation [22]. Therefore, gray target theory appears feasible in predicting or forecasting coal and gas outburst by combining qualitative and quantitative procedures. In addition, the improved AHP method, which is used to determine the weight of the influencing factors of coal and gas outburst, increases the prediction accuracy of the modeling results. 
As described in the Comprehensive hypothesis, coal and gas outbursts are regarded as the result of earth stress, gas pressure, and the physical and mechanical properties of coal. But during the production process in the coal mine, it is difficult to obtain a comprehensive influencing factor value of these three areas. Therefore, often there is a lack of sample data and measurements of influencing factors in coal and gas outburst prediction.

Fortunately, Deng $[14,15]$ presented the gray systems theory to study problems of small samples and poor information. According to a cognitive hierarchy, the research objects are classified into three categories: 'black', 'white', and 'gray'. In this theory, a system is usually defined as a 'black box' if its internal structures and features are completely unknown, whereas 'white' means that the internal features of a system are fully explored. A situation in which the internal features of a system are partly known and partly unknown is called a 'gray system'. In this paper, the gray target model used in the prediction of coal and gas outbursts is just based on gray system theory.

\section{CONCLUSIONS}

In this paper, based on the data measured, gray system theory was used to predict or forecast the coal and gas outburst in the seams in the Jinzhushan coal mine. The situation and forecast contrast were determined, and the following conclusions were drawn based on the actual results of the seam:

1. The use of the gray target modeling in the prediction of coal and gas outbursts, by combining qualitative and quantitative analysis, appears to have more advantages than the use of the fuzzy comprehensive evaluation. The disadvantage of fuzzy comprehensive evaluation is its strong subjectivity and high computational complexity. The method has no uniqueness or symmetry, and implies loss of more information. Contrarily, gray system modeling, a simple and clear procedure, focuses on uncertainty problems as 'small sample', 'poor information', and 'multi-index'. Particularly, for coal and gas outburst involving vague and random factors, the gray target modeling provides a workable approach for its prediction.

2. This study shows that the improved method can calculate the level of the factors affecting the weight. Therefore, the forecast accuracy of gray target modeling is being increased.

3. According to the forecast results, the Jinzhushan coal mine has a high risk of coal and gas outbursts. Protective measures to prevent coal and gas outbursts must be taken in the production process. Gas emission of the coal seam, changes of the coal destruction type, geological structure, and signs of coal and gas outbursts must also be observed.

4. The forecast result obtained by using the gray target model in this paper has a certain guiding significance for production process in Jinzhushan coal mine. In view of that, only four influencing factors were selected in this paper; however, selecting more influencing factors to improve the prediction accuracy is necessary in future research.

\section{ACKNOWLEDGMENTS}

This research was supported in part by the National Natural Science Foundation of China (Grant No. 51304255), the Fundamental Research Funds for the Central Universities (Grant Nos. CDJZR12240055 and CDJZR13240026), the Basic and Frontier Research Projects of Chongqing (Grant No. cstc2013jjB90001), and the Scientific Research Foundation of State Key Laboratory of Coal Mine Disaster Dynamics and Control. 


\section{REFERENCES}

[1] Guo, D.Y., Fan, J.Z., Ma, S.Z. \& Wang, Y.B., Prediction method of coal and gas outburst by analytic hierarchy process and fuzzy comprehensive evaluation. Journal of University of Science and Technology Beijing, 29(7), pp. 660-664, 2007.

[2] You, W., Liu, Y.X., Li, Y., Liu, C.H. \& Zhou, J.B., Predicting the coal and gas outburst using artificial neural network. Journal of China Coal Society, 32(3), pp. 285-287, 2007.

[3] Sun, Y., Yang, S.Q., Wang, B. \& Chu, T.X., Coal and gas outburst forecast by ANN and grey correlation. Journal of Safety Science and Technology, 4(3), pp. 14-17, 2008.

[4] State Administration of Work Safety of People's Republic of China, The Control of Coal and Gas Outburst Regulations, China Coal Industry Publishing House: Beijing, 2009.

[5] Alexeeva, A.D., Revva, V.N., Alyshev, N.A. \& Zhitlyonok, D.M., True triaxial loading apparatus and its application to coal outburst prediction. International Journal of Coal Geology, 58(4), pp. 245-250, 2004. doi: http://dx.doi.org/10.1016/j.coal.2003.09.007

[6] Kadsuo, K., Basic study on analyzing occurring mechanism of gas outburst. Coal Mine Technology, 1, pp. 16-19, 1980.

[7] Sommerton, W.J., Soylemezoglu, I.M. \& Dudley, R.C., Effect of stress on permeability of coal. International Journal of Rock Mechanics and Mining Sciences and Geomechanics, 12(2), pp. 129-145, 1975. doi: http://dx.doi.org/10.1016/01489062(75)91244-9

[8] Peng, S.J., Xu, J., Yang, H.W. \& Liu, D., Experimental study on the influence mechanism of gas seepage on coal and gas outburst disaster. Safety Science, 50(4), pp. 816-821, 2012. doi: http://dx.doi.org/10.1016/j.ssci.2011.08.027

[9] Peng, S.J., Xu, J., Yin, G.Z., Liu, D. \& Wang, W.Z., Spatial-temporal evolution of gas migration pathways in coal during shear loading. International Journal of Mining Science and Technology, 22(6), pp. 769-773, 2012. doi: http://dx.doi.org/10.1016/j. ijmst.2012.11.009

[10] Wu, A.Y. \& Xiao, H.F., Wang, C.L. \& He, L.W., Establishment and application of weights and gray association model based on coal and gas outburst controlled factors assessment. Journal of China Coal Society, 30(1), pp. 58-62, 2005.

[11] Zhang, F.Y. \& Han, Y., Application of grey relevancy analysis method in index optimization of outburst forecast when a cross-cut is uncovered. Journal of China Coal Society, 32(10), pp. 1023-1025, 2007.

[12] Han, Y.P., Improved grey correlative model for risk assessment on coal and gas outburst. Journal of Henan Polytechnic University, 29(3), pp. 316-319, 2010.

[13] Liang, B., Qin, B. \& Sun, W.J., Possibility assessment of coal-gas outburst based on grey target model. Journal of China Coal Society, 36(12), pp. 1974-1978, 2011.

[14] Deng, J.L., Control problems of grey systems. Systems and Control Letters, 1(5), pp. 288-294, 1982. doi: http://dx.doi.org/10.1016/s0167-6911(82)80025-x

[15] Deng, J.L., Grey System Fundamental, Huazhong University of Science \& Technology Press: Wuhan, 2002.

[16] Chen, S.W., Li, Z.G. \& Xu, Q.S., Grey target theory based equipment condition monitoring and wear mode recognition. Wear, 260, pp. 438-449, 2005. doi: http://dx.doi. org/10.1016/j.wear.2005.02.085

[17] Xu, J. \& Yao, G., Research of factors affecting the agro products logistics based on the AHP - grey correlation analysis. INMATEH - Agricultural Engineering, 40(2), pp. 19-26, 2014. 
[18] Xiong, H., Sun, C.X., Du, P., Dai, Y. \& Wang, Q., Synthetic assessment of power transformer condition based on Matter-Element theory. Journal of Chongqing University (Natural Science Edition), 29(10), pp. 24-27, 2006.

[19] Li, B., Wei, J. \& Li, P., Nonlinear motion law of coal-bed gas seepage under the combined effects of stress and temperature. Journal of Power Technologies, 94(2), pp. 1-8, 2014.

[20] Macian-Martinez, V., Tormos-Martinez, B., Gomez-Estrada, Y.A. \& Bermudez-Tamarit, V., A review of degradation process on compressed natural gas and diesel engines lubricant oils. DYNA, 88(1), pp. 49-58, 2013.

[21] Abhay, M.V. \& Tushar, M., Coal bed methane exploration: a journey from alternative energy option to the environment polluting agent. Nature Environment and Pollution Technology, 9(3), pp. 575-580, 2010.

[22] Liu, L.M. \& Xiao, H.F., Application of neural network to predict coal and gas outburst. Mining Safety \& Environmental Protection, 30(1), pp. 34-37, 2003. 